\title{
WHITNEY SINGULARITIES OF SOLUTIONS OF PARTIAL DIFFERENTIAL EQUATIONS
}

\author{
Alexander B. GiventAL
}

\begin{abstract}
We classify singularities of multiple-valued solutions of higher order systems of partial differential equations in the case when projections of the corresponding integral manifolds of the Cartan distribution in the jet space to the space of independent variables have Whitney singularities.
\end{abstract}

\section{$\S 0$ InTRODUCTION}

A propagating wave front of geometrical optics, initially non-singular, can become singular. This property of geometrical optics can be interpreted as that of solutions of Hamilton - Jacobi equations to be multiple-valued even if the initial data of the Cauchy problem are single-valued. The fronts are in fact levels of such a solution, the initial front may be viewed as a source of the light propagation, and if a few different rays from the source deliver light to a given target point, their optical lengths are exactly the few values of the solution at this point.

A systematic study of ramifications of solutions of Hamilton - Jacobi equations by means of singularity theory was begun by V.Arnold in 1972 and led him to a remarkable theory [1], [4] of singularities of wave fronts and caustics and to the discovery of their relations with the discriminants of reflection groups.

The success of Arnold's approach created for successors a good pattern to follow. The approach is based on the fact that in geometrical optics the differential of a solution of a Hamilton-Jacobi equation describes a non-singular submanifold in the cotangent bundle of the domain. It is in fact a Lagrangian submanifold of the cotangent bundle located within the hypersurface given by the Hamilton - Jacobi equation, and the solution ramifies exactly where the projection of the submanifold to the domain degenerates. The idea now is to forget about the Hamilton - Jacobi equation and to classify typical projections of Lagrangian submanifolds from the cotangent bundle to the base. An answer to this problem would also describe singularities of solutions of generic Hamilton - Jacobi equations (since a hypersurface containing the Lagrangian submanifold can be always perturbed along with it) while the classification of singularities for a given Hamilton - Jacobi equation constitutes a separate problem.

In the general context of partial differential systems Hamilton - Jacobi equations form a special class of 1-st order PDEs on one unknown function. A more general problem - classification of singularities of multiple-valued vector-solutions for systems of the 1-st order PDEs - was considered by V.Lychagin [12]. Following to

This research is partially supported by NSF grant DMS-90-01089 and by Alfred P. Sloan Foundation 
the pattern above one should interpret partial differential equations as algebraic relations between functions and their derivatives in a jet space of vector-functions. The jet spaces bear canonical Cartan distributions expressing the fact that the variables standing for derivatives of functions are really the derivatives of the variables standing for the functions themselves. A (multiple-valued, or generalized) solution of a PDE system is an integral submanifold of the Cartan distribution in the jet space, assumed to be non-singular and located on the submanifold determined by the PDE system in question. Bearing in mind applications to generic PDE systems one drops the system itself and simply classifies the singularities of multiple-valued (vector-)functions as those of projections of such integral submanifolds from the jet space to the base.

It is not true in general that generic phenomena for projections of integral manifolds are the same as for generic mappings of manifolds of equal dimensions. However, if a stable singularity of differential mappings can be realized as a projection of an integral submanifold then perturbations of the integral submanifold (within the class of integral submanifolds) realize the same singularity type of the projection to the base. It turns out that this idea applies pretty well to so called Whitney singularities, that is generic maps $\mathbb{R}^{n} \rightarrow \mathbb{R}^{n}$ with the differential of corank $\leqslant 1$. Their classification form a discrete series, $n=1,2,3, \ldots$ (fold, cusp, . .) and they all can be realized by integral submanifolds.

It is where the idea of Lychagin's paper comes from. He fixes a Whitney type germ of the projection to the base and classifies generic integral embeddings of the projected manifold into the 1-jet space of vector-functions over the given projection to the base. The classification turns out to be discrete in the case where the number of independent variables of the vector-functions in question does not exceed their vector dimension.

Later [6] I interpreted the result and the normal forms of the singularities geometrically. Singularities (that is ramification) of multiple-valued functions are better displayed by their graphs - multiple-valued "sections" of the 0-jet bundle. The graphs of Lychagin's singularities turn out to be isomorphic to so called open swallowtails - the strata of the discriminants in spaces of polynomials in one variable where the polynomial has a root of multiplicity exceeding half of its degree. These varieties first arose probably in Hilbert's invariant theory of binary forms and then occurred in various singularity problems $([2],[7])$ in particular in the role of Lagrangian varieties in geometrical optics on manifolds with boundary ([3], [ 8]).

The purpose of the present paper is to extend this geometrical approach to the general case of systems of higher order PDE. Following V.Arnold we begin with a non-singular $N$-dimensional integral submanifold $X$ of the Cartan distribution in the $m$-jet space of $d$-dimensional vector-functions of $N$ variables, and "forget" the differential equations (which are considered to be generic). Then following V.Lychagin we assume the projection of the integral submanifold to the base to be Whitnean, of index $n$ in the classification of corank- 1 singularities of differential maps (see §1). The idea of our geometrical approach is based on construction of universal varieties for Whitney maps. This means that we begin with studying arbitrary " $m$ times differentiable" multiple-valued scalar functions with fixed projection of the corresponding integral submanifold to the base. All such functions lifted to $X$ generate there a subalgebra in the algebra of regular functions. This subalgebra can be viewed as the algebra of regular functions on some variety $\Gamma_{m}^{n}$ parametrized by $X$. By the very construction, this variety has the universal 
property that whenever $X$ is embedded (over the given projection to the base) into an $m$-jet space as an integral submanifold of the Cartan distribution, its map to the 0-jet space onto the graph of the corresponding multiple-values section factors through $\Gamma_{m}^{n}$. In particular if the embedded dimension of $\Gamma_{m}^{n}$ does not exceed the dimension $N+d$ of the 0 -jet space the graphs should be, generically, isomorphic to $\Gamma_{m}^{n}$. Studying further the universal varieties of Whitnean mappings we will show that they are straightforward "higher derivative" generalizations of open swallowtails and that their position in the 0-jet space can be brought to a normal form by suitable equivalence transformations.

In order to describe here the normal forms we have to introduce some discriminants in spaces of polynomials with multiple critical points.

Let us consider at first the space of polynomials in one variable, $x$, of degree $m(n+1)+1$ whose derivative has $n+1$ roots of multiplicity $m$. Such polynomials can be written in the form (we fix the 1-st coefficient and reduce the root sum to $0)$

$$
\int_{0}^{x}\left(y^{n+1}+q_{1} y^{n-1}+\cdots+q_{n}\right)^{m} d y+u
$$

where $\left(q_{1}, \ldots, q_{n}, u\right)$ are arbitrary coefficients. Let $\Delta_{m-1}^{n}$ denote the discriminant in this polynomial space that is the hypersurface of polynomials with multiple roots (the multiplicity jumps automatically to $\mathrm{m}+1$ so that only $\Delta_{0}^{n}$ is the usual swallowtail - the discriminant in the space of all polynomials of degree $n+2)$. This hypersurface can be considered as the graph of a multiple-valued (in fact $n+1$ valued) scalar function $u$ of $n$ independent variables $q$. The graphs with $n=2$ are shown in Figure 1. It displays a series of 3-valued functions with growing "number of derivatives" to be well-defined (topologically the surfaces alternate between the first two). These functions will serve us as normal forms in the "higher derivative" generalization of Arnold's scalar problem.

In order to describe normal forms in "higher Lychagin's problem" let us consider the space of polynomials of degree $m(n+1)+n$ with the property that their $n$-th derivative has $n+1$ roots of multiplicity $m$ :

$$
\begin{gathered}
\int_{0}^{x} d y_{n} \int_{0}^{y_{n}} \cdots \int_{0}^{y_{2}}\left(y_{1}^{n+1}+\cdots+q_{n}\right)^{m} d y_{1} \\
+u_{1} x^{n-1}+\cdots+u_{n}
\end{gathered}
$$

where now the arbitrary coefficients are $\left(q_{1}, \ldots, q_{n}, u_{1}, \ldots, u_{n}\right)$. In the discriminant of this polynomial space let us consider the closed stratum, denoted $\Gamma_{m}^{n}$, of the polynomials with a root of multiplicity $>n$ (it automatically jumps to $n+m$ at least). This stratum is an $n$-dimensional variety in the $2 n$-dimensional space and can be considered as the graph of the $n+1$-valued vector-function $\left(u_{1}, \ldots, u_{n}\right)$ of $n$ independent variables $q$. One can check that this vector-function is the gradient of the scalar function described by the graph $\Delta_{m}^{n}$ in the previous paragraph. On one hand this shows that Figure 1 accumulates information on these vector-functions as well. On the other hand this means that $\Gamma_{m}^{n}$ have Lagrangian embeddings into $T^{*} \mathbb{R}^{n}$ and explains that "strange" circumstance that in fact they have already occurred in the Lagrangian role in a paper by V.Zakalyukin [16] (this paper contains a generalization of [ 8] where Lagrangian open swallowtails $\Gamma_{1}^{n}$ arose).

In the following formulation of our main theorem we call standard the germs at the origin of the multiple-valued (vector-)functions described above in terms of discriminants in the polynomial spaces. 


\section{Theorem.}

1(scalar problem) A germ of a multiple-valued function at a point where the projection of its $m$-jet extension to the space of independent variables has a Whitney singularity of index $n$ is equivalent to the standard one with the graph $\Delta_{m-1}^{n}$.

2 (vector problem) A germ of a generic multiple-valued $m$ times differentiable $d$ dimensional vector-function at a generic point among those where the projection of its m-jet extension has the Whitney singularity of index $n$ is equivalent to the standard one with the graph $\Gamma_{m}^{n}$ provided that $n \leqslant d$.

The equivalence of multiple-valued functions in this theorem involves compositions of the following operations:

In the scalar case - change of independent variables, addition of new independent variables on which the function does not depend explicitly, reversal of the function's sign and addition with a single-valued regular [Afunction of independent variables.

In the vector case - change of independent variables, addition of new independent variables, addition of new dependent variables (that is additional zero components to the vector-function) and fiberwise-affine changes of dependent variables with coefficients which are regular functions of independent variables.

The proof of the theorem is given in $\S 5$ after more detailed definitions given in $\S 1$ and $\S 2$ and some preliminary results of $\S 3$ and $\S 4$ which also contain important extra information on the normal forms and universal varieties. In particular we will see that $k$-jet extensions of $\Gamma_{m}^{n}$ with $0<k<m$ are equivalent as multiple-valued vector-functions to $\Gamma_{m-k}^{n}$.

The main theorem equally holds in smooth, real-analytic and complex-analytic categories. We prefer to work in the real-analytic set-up. The complex case differs only by notations. The only point where we use analyticity is our elementary proof of the main technical lemma (the versality theorem for fractional forms). A proof of this lemma in the smooth case can be found in the literature [10].

\section{$\S 1$ Whitney Singularities}

In the space $\mathbb{R}^{n+1}$ with coordinates $x, q_{1}, \ldots, q_{n}$, let us consider a hypersurface given by the equation $F_{n}=0$ where

$$
F_{n}(x, q)=x^{n+1}+q_{1} x^{n-1}+\cdots+q_{n-1} x+q_{n} .
$$

The hypersurface is isomorphic to $\mathbb{R}^{n}$. Its projection $(x, q) \mapsto q$ to the space $\mathbb{R}^{n}$ with coordinates $q_{1}, \ldots, q_{n}$ is called Whitney mapping (see Figure 2 where $n=2$ ). At the origin the Whitney mapping has a corank-1 singularity that is its differential degenerates and has rank next to the maximal one.

The Whitney mappings serve as normal forms for generic corank-1 singularities of differential maps $\mathbb{R}^{n} \rightarrow \mathbb{R}^{n}$. Two germs of such maps are called (left-right) equivalent if they can be transformed into one another by suitable germs of diffeomorphisms in the source and target spaces.

Theorem (B.Morin [ 13], [ 9], [4]). A germ of a generic differential map between manifolds of the same dimension at any point of corank 1 is equivalent to the germ at the origin of a Whitney map or its suspension.

By the suspension of a map we mean its cartesian product with the identity map $\mathbb{R}^{k} \rightarrow \mathbb{R}^{k}$. In particular, the theorem implies that the Whitney mapping to $\mathbb{R}^{n}$ near 
any point is locally equivalent to such a suspension of one of the Whitney mappings to $\mathbb{R}^{n-k}$ near the origin.

\section{$\S 2$ Cartan distributions}

Let $J^{k}(n, d)$ denote the $k$-jet space of sections of a bundle over $\mathbb{R}^{n}$ with the fiber $\mathbb{R}^{d}$. We will usually assume that the bundle is provided with an affine structure. Introducing coordinates $\left(q_{1}, \ldots, q_{n}\right)$ on the base and (affine) coordinates $\left(p_{1}, \ldots, p_{d}\right)$ on the fiber induces a coordinate system $\left(q_{j}, p_{i}^{\alpha}\right)$ on $J^{m}(n, d)$ where $j=1, \ldots, n$, $i=1, \ldots, d$, and $\alpha=\left(\alpha_{1}, \ldots, \alpha_{n}\right), \alpha_{j} \geqslant 0$, is a multi-index of degree $|\alpha|=\alpha_{1}+$ $\cdots+\alpha_{n} \leqslant m$. Here $p_{i}^{\alpha}$ stands for the partial derivative

$$
\frac{\partial^{|\alpha|} p_{i}}{\partial q_{1}^{\alpha_{1}} \ldots \partial q_{n}^{\alpha_{n}}}
$$

so that $p_{i}=p_{i}^{0, \ldots, 0}$.

The $k$-jet spaces form the tower of affine bundles,

$$
\mathbb{R}^{n} \leftarrow J^{0}(n, d) \leftarrow \cdots \leftarrow J^{m}(n, d) \leftarrow \cdots
$$

where the arrows mean "forgetting the highest derivatives".

Given a section $p=p(q)$ of the bundle $J^{0}(n, d)$, its $m$-jet extension is defined by putting $p_{i}^{\alpha}=\partial^{|\alpha|} p_{i}(q) / \partial q^{\alpha}$. It is a section of the bundle $J^{m}(n, d)$ integral to the Cartan distribution. The latter is given by the coordinate formulas

$$
d p_{i}^{\alpha}=\sum_{j} p_{i}^{\alpha+1_{j}} d q_{j},|\alpha|<m, i=1, \ldots, d
$$

Vice versa, an integral section of the Cartan distribution on $J^{m}(n, d)$ is the $m$-jet extension of its projection to $J^{0}(n, d)$.

Definition. A multiple-valued $m$ times differentiable vector-function on $\mathbb{R}^{n}$ is an integral $n$-dimensional submanifold $X$ of the Cartan distribution on $J^{m}(n, d)$ transversal to the fibers of the projection $J^{m}(n, d) \rightarrow \mathbb{R}^{n}$ almost everywhere. The graph of the multiple-valued function is the image $X^{(0)}$ of $X$ under the projection $J^{m}(n, d) \rightarrow J^{0}(n, d)$ while the images $X^{(k)}$ in $J^{k}(n, d), k=0, \ldots, m$ are called $k$-jet extensions of the function.

According to this definition a multiple-valued function is locally just a vectorfunction (section) almost everywhere. It can be recovered by its graph as the closure of the union of the $m$-jet extensions of its single-valued branches.

Two (germs of) multiple-valued functions are called equivalent if their graphs can be transformed into one another by (the germ of) a diffeomorphism of the space $J^{0}(n, d)$ preserving fibers of the projection to the base $\mathbb{R}^{n}$ and affine on each fiber. Such a diffeomorphism lifts canonically to a fiberwise-affine diffeomorphism of $J^{m}(n, d)$ preserving the Cartan distribution and transforming the corresponding integral submanifolds into one another (so that the reader can reformulate the definition of equivalence in terms of the integral submanifolds only).

\section{$\S 3$ LAGRANGIAN MODELS}

Now we introduce and study the varieties that will serve as normal forms for graphs of multiple - valued functions (solutions of PDE). However it is convenient 
to describe these varieties as Lagrangian or Legendrian ones with respect to the symplectic or contact structure in the ambient space which seems to have little to do with the PDE in question.

Let $\left(q_{1}, \ldots, q_{n}, p_{1}, \ldots, p_{n}\right.$ (, and may be $\left.u\right)$ ) be Darboux coordinates in the standard symplectic (respectively contact) space $\mathbb{R}^{2 n(+1)}$. This means that the symplectic (contact) structure is given by the formula

$$
\begin{aligned}
& d p_{1} \wedge d q_{1}+\cdots+d p_{n} \wedge d q_{n} \\
& \left(d u=p_{1} d q_{1}+\cdots+p_{n} d q_{n}\right) .
\end{aligned}
$$

A subset $L \subset \mathbb{R}^{2 n(+1)}$ which is (diffeomorphic to) an algebraic variety is called Lagrangian (respectively Legendrian) if 1) each irreducible component of $L$ has dimension $n$ and 2) it is isotropic (integral to the contact structure, respectively) at every non-singular point. "Forgetting $u$ ", obviously, projects Legendrian varieties to Lagrangian ones unless all the fibers are positive - dimensional.

Let $G(x, q)$ be a family of functions of $x$ parametrized by $q$. One call $G$ generating family of the Lagrangian (Legendrian) variety $L$ if

$$
L=\left\{(q, p(, u)) \mid \exists x: \frac{\partial G}{\partial x}(x, q)=0, p=\frac{\partial G}{\partial q}(x, q)(, \text { and } u=G(x, q))\right\} .
$$

Definition. We denote $\Gamma_{m}^{n}$ the Legendrian variety in $\mathbb{R}^{2 n+1}=J^{1} \mathbb{R}^{n}=\mathbb{R} \times T^{*} \mathbb{R}^{n}$ generated by the family of functions in one variable, $x$ :

$$
G_{m, n}(x, q)=\int_{0}^{x} F_{n}^{m+1}(y, q) d y
$$

We will see soon that it projects isomorphically onto a Lagrangian variety in $\mathbb{R}^{2 n}=$ $T^{*} \mathbb{R}^{n}$. Therefore we may keep the same notation $\Gamma_{m}^{n}$ for the Lagrangian variety determined by the generating family $G$.

Our objective now is to describe the algebra $\mathcal{O}_{\Gamma_{m}^{n}}$ of regular functions on the variety $\Gamma_{m}^{n}$. Let us notice at first that $\Gamma_{m}^{n}$ has a non-singular normalization $\mathbb{R}^{n}$, namely it is parametrized by the variables $\left(x, q_{1}, \ldots, q_{n-1}\right)$, and the composition $\mathbb{R}^{n} \rightarrow T^{*} \mathbb{R}^{n} \rightarrow \mathbb{R}^{n}$ of the parametrization map with the projection map $(p, q) \mapsto$ $q=\left(q_{1}, \ldots q_{n}\right)$ is exactly the canonical Whitney mapping. The algebra $\mathcal{O}_{\mathbb{R}^{n}}=$ $\mathbb{R}\left\{x, q_{1}, \ldots, q_{n-1}\right\}$ of regular functions on the parametrizing space can be viewed as a module over the algebra $\mathbb{R}\{q\}$ of regular functions on the target space, and for the Whitney mapping this module is free of rank $n+1$ with generators $\left(1, x, x^{2}, \ldots, x^{n}\right)$. This follows from the Weierstrass preparation theorem which says that any function $\phi(x, q)$ can be represented in the form

$$
\phi(x, q)=H(x, q) F_{n}(x, q)+Q_{1}(q) x^{n}+\cdots+Q_{n}(q) .
$$

Theorem 1. 1) The algebra $\mathcal{O}_{\Gamma_{m}^{n}}$ is the subalgebra in $\mathcal{O}_{\mathbb{R}^{n}}$ of all functions of the form

$$
\int_{0}^{x} \Phi(y, q) F_{n}^{m}(y, q) d y+Q(q)\left(\bmod F_{n}\right) .
$$

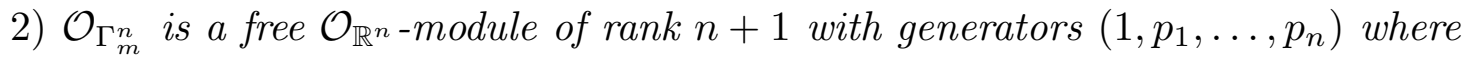

$$
p_{j}=\frac{\partial G_{m, n}}{\partial q_{j}} \sim \int^{x} y^{n-j} F_{n}^{m}(y, q) d y .
$$


Proof. At first, functions of the form $\int \Phi F_{n}^{m} d y+Q\left(\bmod F_{n}\right)$ form a subalgebra in $\mathcal{O}_{\mathbb{R}^{n}}$. This is proved by "integration by parts":

$$
\frac{\partial}{\partial x}\left(\int^{x} \Phi_{1} F_{n}^{m} d y\right)\left(\int^{x} \Phi_{2} F_{n}^{m} d y\right)
$$

is divisible by $F_{n}^{m}$.

On the other hand, this subalgebra obviously contains all $q_{i}, p_{j}$ and $u=\int F_{n}^{m+1} d y$ and therefore contains the algebra $\mathcal{O}_{\Gamma_{m}^{n}}$ generated by these functions. Thus it suffices to prove that any function of the form $\int \Phi F_{n}^{m} d y+Q\left(\bmod F_{n}\right)$ belongs to the submodule generated by $\left(1, p_{1}, \ldots, p_{n}\right)$.

Lemma. For any function $\Phi(x, q)$ the product $\Phi F_{n}^{m}$ can be represented in the form

$$
\Phi(x, q) F_{n}^{m}(x, q)=\frac{\partial}{\partial x}\left(H(x, q) F_{n}^{m+1}(x, q)\right)+\sum_{1}^{n} Q_{j}(q) x^{n-j} F_{n}^{m}(x, q) .
$$

We delay the proof of this lemma till the end of this section.

Integrating this representation gives rise to

$$
\begin{aligned}
& \int_{0}^{x} \Phi(y, q) F_{n}^{m}(y, q) d y \bmod F_{n}(x, q) \\
& =-H(0, q) F_{n}^{m+1}(0, q)+\frac{1}{m+1} \sum_{j=1}^{n} Q_{j}(q) p_{j} .
\end{aligned}
$$

Corollary 1. The Lagrangian and Legendrian varieties generated by the family $G_{m, n}$ are isomorphic.

Now we will treat the Lagrangian variety $\Gamma_{m}^{n}$ as the graph of the differential of an $(n+1)$-valued function of the variables $\left(q_{1}, \ldots, q_{n}\right)$. This function, denoted $U_{m, n}=U_{m, n}(q)$, is given by its graph - a hypersurface in $J^{0} \mathbb{R}^{n}=\mathbb{R}^{n} \times \mathbb{R}$. This hypersurface, denoted $\Delta_{m}^{n}$, is obtained from Legendrian $\Gamma_{m}^{n}$ by projecting $(q, p, u) \mapsto(q, u)$. It can be interpreted as the discriminant in a suitable space of polynomials, namely the polynomials of degree $(n+1)(m+1)+1$ in one variable, $x$, whose all extrema have orders multiple to $m+1$ (and with additional restrictions that the top coefficient is fixed and the next one equals 0 (see Figure 1).

Corollary 2. The multiple-valued function $U_{m, n}$ is differentiable $m+1$ times.

By definition, this means that all the partial derivatives of all branches of this function extend to functions regular on the normalization $\mathbb{R}^{n}$ of the graph $\Delta_{m}^{n}$. For first derivatives this is true by the very definition of $\Delta_{m}^{n}$ since they extend to $\left(p_{1}, \ldots, p_{n}\right)$.

Proof. Induction by the order of derivatives and the formula

$$
\begin{aligned}
\left.\frac{\partial}{\partial q_{j}}\right|_{F_{n}=0} \int^{x} \Phi F_{n}^{k} d y & =-\frac{x^{n-j}}{F_{n}^{\prime}} \Phi F_{n}^{k}+ \\
+\frac{\partial}{\partial q_{j}} \int^{x} \Phi F_{n}^{k} d y & =\left(\bmod F_{n}\right) \int^{x} \Psi F_{n}^{k-1} d y .
\end{aligned}
$$


Corollary 3. The differential of the multiple-valued function $U_{m, n}$ considered as a multiple-valued vector-function $p=p(q)$ given by its graph - Lagrangian $\Gamma_{m}^{n}$, is $m$ times differentiable.

Let us consider now the $k$-jet extension of the function $U_{m, n}$. Its graph - a variety in the $k$-jet space of functions on $\mathbb{R}^{n}$, has the algebra of regular functions generated by $q$ 's, $u$ and all its partial derivatives of orders $\leqslant k$. These algebras with $k=0, \ldots, m+1$ form a tower

$$
\mathcal{O}^{(0)} \subset \mathcal{O}^{(1)} \subset \cdots \subset \mathcal{O}^{(m+1)} \subseteq \mathcal{O}_{\mathbb{R}^{n}}
$$

Corollary 4. The $k$-jet extension varieties of $\Delta_{m}^{n}$ are isomorphic to $\Gamma_{r}^{n}$ with $r=$ $m+1-k$ for $k=1, \ldots, m+1$. In particular the tower of the jet extension varieties coincides with

$$
\Delta_{m}^{n} \leftarrow \Gamma_{m}^{n} \leftarrow \Gamma_{m-1}^{n} \leftarrow \cdots \leftarrow \Gamma_{1}^{n} \leftarrow \Gamma_{0}^{n}=\mathbb{R}^{n}
$$

Remark. Notice that the $m+1$-jet extension variety is the non-singular normalization of all the preceeding varieties in this tower. The $m$-jet extension variety is exactly the "open swallowtail" that has been studied in [6], [7], [8] in connection with geometrical optics of diffraction and Lychagin's singular solutions of the 1-st order PDE.

Proof. From the inductive proof of Corollary 3 one can see that all partial derivatives of $U_{m, n}$ up to order $k=m+1-r$ belong to the algebra of regular functions on $\Gamma_{r}^{n}$. On the other hand the derivatives $\partial^{k} U_{m, n} / \partial q_{n}^{k-1} \partial q_{j}$ are proportional to

$$
\int^{x} y^{n-j} F_{n}^{r} d y, j=1, \ldots, n
$$

and thus generate the algebra of regular functions on $\Gamma_{r}^{n}$ as $\mathbb{R}\{q\}$-module.

Proof of the lemma. Our proof is based on an interpretation of the lemma as the infinitesimal versality condition for fractional forms on a line. Let us consider the differential form

$$
x^{n+1}(d x)^{\alpha}, \alpha=1 /(m+1) .
$$

We claim that the family of such forms

$$
F_{n}(x, q)(d x)^{\alpha}
$$

is its miniversal unfolding. By definition this means that another family, say

$$
\left(F_{n}+\epsilon \Phi\right)(d x)^{\alpha}
$$

can be induced from a family fiberwise equivalent to the first one.

Linearizing in $\epsilon$ we find that any $\Phi(d x)^{\alpha}$ can be represented as Lie derivative of $F_{n}(d x)^{\alpha}$ along a vector field of the form

$$
H(x, q) \frac{\partial}{\partial x}+Q_{1}(q) \frac{\partial}{\partial q_{1}}+\cdots+Q_{n}(q) \frac{\partial}{\partial q_{n}}
$$


Multiplying this relation by $F_{n}^{m}$ we come to the statement of the lemma:

$$
(m+1) \Phi F_{n}^{m} d x=d_{x}\left(H F_{n}^{m+1}\right)+\sum Q_{j} \partial F_{n}^{m+1} / \partial q_{j} .
$$

Our claim can be justified by the references [10], [ 11], [ 12] to more general versality theorems. For the sake of completeness we reproduce here our original proof [ 8] that works exactly for $\alpha=1 /(m+1)$ in the complex or real-analytic categories. It consists in reducing the versality statement for fractional forms to the well-known versality theorem for functions [9], [4]. Let us consider the following family of functions in one variable:

$$
\int^{x}\left(F_{n}+\epsilon \Phi\right)^{m+1} d y
$$

It is an unfolding of the function $x^{D+1} /(D+1)$ with $D=(n+1)(m+1)$. By the versality theorem for function's singularities this unfolding can be induced from a family equivalent to

$$
\begin{gathered}
x^{D+1} /(D+1)+\lambda_{1} x^{D-1} /(D-1)+\cdots+\lambda_{D-1} x \\
=\int^{x}\left(y^{D}+\cdots+\lambda_{D-1}\right) d y .
\end{gathered}
$$

The crucial observation is that the original family consists of the functions whose all extrema have orders multiple to $m+1$, and this property is invariant under changes of the variable. Therefore the family is actually equivalent to one induced from the subfamily of those polynomials of degree $D+1$ whose extrema satisfy this condition. In the analytic case this subfamily is non-singular and coincides with

$$
\int^{x} F_{n}^{m+1} d y
$$

Passing to differentials of functions (and taking $m+1$-st root) completes the proof.

\section{$\S 4$ UNIVERSAL VARIETIES}

Our objective now is to study germs of multiple-valued functions at the points where the projection $X \rightarrow \mathbb{R}^{n}$ of the integral submanifold to the base has a Whitney singularity. This means that in suitable local coordinate systems on $X$ and $\mathbb{R}^{n}$ the projection is given by the standard formulas

$$
\left(x, q_{1}, \ldots, q_{n-1}\right) \mapsto\left(q_{1}, \ldots, q_{n-1}, q_{n}=-\left(x^{n+1}+\cdots+q_{n-1} x\right)\right) .
$$

Thus it makes sense to talk about subalgebras $\mathcal{O}_{\Gamma_{k}^{n}}$ in the algebra of germs of regular functions on $X$ at such a point.

The embedded dimension of $\Gamma_{m}^{n}$ equals $2 n$. In the algebraic tangent $2 n$ - dimensional space to $\Gamma_{m}^{n}$ at the origin there is a complete flag

$$
T^{0} \subset T^{1} \subset \cdots \subset T^{2 n}
$$

intrinsically related to $\Gamma_{m}^{n}$. In the canonical coordinates (q,p) of the Lagrangian model for $\Gamma_{m}^{n}$ the subspace $T^{k}$ is given by the equations

$$
\begin{gathered}
p_{1}=\cdots=p_{2 n-k}=0 \text { for } k \geqslant n \\
p_{1}=\cdots=p_{n}=q_{n}=\cdots=q_{k+1}=0 \text { for } k<n .
\end{gathered}
$$


Theorem 2. The correspondence between multiple-valued functions and their graphs transforms germs of multiple - valued functions $X \hookrightarrow J^{m}(n, d)$ with Whitnean projection to the base to germs of mappings $\Gamma_{m}^{n} \rightarrow J^{0}(n, d)$ with non - degenerate restriction of its differential to the subspace $T^{n+1}$.

Remark. This theorem displays the universal role that $\Gamma_{m}^{n}$ plays for the multiplevalued vector-functions in question.

Proof of the theorem.

Lemma 1. Let $p_{i}^{\alpha}\left(x, q_{1}, \ldots, q_{n-1}\right)$ be the pullback to $X$ of the coordinate function $p_{i}^{\alpha}$ on the jet space $J^{m}(n, d)$. Then

$$
p_{i}^{\alpha}(x, q)=\frac{\partial^{|\alpha|}}{\partial q^{\alpha}}\left[Q_{i}(q)+\int_{0}^{x} \frac{\partial p_{i}^{(0, \ldots, 0, m)}}{\partial x}(y, q) \frac{F_{n}^{m}(y, q)}{m !} d y\right]
$$

where $Q_{i}$ are some regular functions of $q$.

Proof. From the Cartan relation $d p^{\alpha}=\cdots+p^{\alpha+1_{n}} d q_{n}$ we have

$$
p^{\beta} F^{\prime}=-\left(p^{\beta-1_{n}}\right)^{\prime}, p^{\beta-1_{n}} F^{\prime}=-\left(p^{\beta-2_{n}}\right)^{\prime}, \ldots
$$

For $k \leqslant \beta_{n}$ integration by parts gives:

$$
\begin{aligned}
\frac{1}{k !} & \int_{0}^{x}\left(p^{\beta}\right)^{\prime} F^{k} d y= \\
& =\left.p^{\beta} \frac{F^{k}}{k !}\right|_{0} ^{x}-\frac{1}{(k-1) !} \int_{0}^{x} p^{\beta} F^{\prime} F^{k-1} d y \\
& =\left.p^{\beta} \frac{F^{k}}{k !}\right|_{0} ^{x}+\frac{1}{(k-1) !} \int_{0}^{x}\left(p^{\beta-1_{n}}\right)^{\prime} F^{k-1} d y \\
& =\left.p^{\beta} \frac{F^{k}}{k !}\right|_{0} ^{x}+\left.p^{\beta-1_{n}} \frac{F^{k-1}}{(k-1) !}\right|_{0} ^{x}-\frac{1}{(k-2) !} \int_{0}^{x} p^{\beta-1_{n}} F^{\prime} F^{k-2} d y \\
& =\cdots \\
& =\left.\sum_{s=0}^{k} p^{\beta-s_{n}} \frac{F^{k-s}}{(k-s) !}\right|_{0} ^{x} .
\end{aligned}
$$

This implies

$$
p_{i}^{\alpha}(x, q)=\sum_{s=0}^{k} p_{i}^{\alpha+s_{n}}(0, q) \frac{q_{n}^{s}}{s !}+\frac{1}{k !} \int_{0}^{x} \frac{\partial p_{i}^{\alpha+k_{n}}}{\partial x}(y, q) F_{n}^{k}(y, q) d y .
$$

In particular

$$
p^{(0, \ldots, 0)}=Q(q)+\int_{0}^{x}\left(p^{(0, \ldots, 0, m)}\right)^{\prime} \frac{F^{m}}{m !} d y
$$

where $Q$ is regular. From the Cartan relations and $F(x, q)=0$ we get

$$
p^{\alpha+1_{j}}=\frac{\partial p^{\alpha}}{\partial q_{j}}-\frac{x^{n-j}}{F^{\prime}} \frac{\partial p^{\alpha}}{\partial x} .
$$


(Here we consider $p^{\alpha}$ as a function of the variables $\left(x, q_{1}, \ldots, q_{n-1}, q_{n}\right)$ constrained by $F(x, q)=0$ only after the partial derivatives are calculated.) Applying this inductively we obtain

$$
p^{\alpha}=\frac{\partial^{|\alpha|}}{\partial q^{\alpha}}\left(Q(q)+\int_{0}^{x}\left(p^{(0, m)}\right)^{\prime} \frac{F^{m}}{m !} d y\right)
$$

since the 'second term', involving the derivative in $x$, disappears due to the constraint.

Corollary 1. Let $X \subset J^{m}(n, d)$ be a germ of a multiple-valued function with the Whitney singularity of the projection to the base. Then regular functions on its $(m-k)$-jet extension $X^{(m-k)},(k=0, \ldots, m)$, form a subalgebra in $\mathcal{O}_{\Gamma_{k}^{n}}$.

Corollary 2. The tower

$$
X=X^{(m)} \rightarrow \cdots \rightarrow X^{(0)} \rightarrow \mathbb{R}^{n}
$$

of jet extensions of a multiple-valued function germ at a point with the Whitney projection to the base is the image of the tower

$$
\Gamma_{0}^{n} \rightarrow \cdots \rightarrow \Gamma_{m}^{n} \rightarrow \mathbb{R}^{n}
$$

under a suitable tower mapping to

$$
J^{m}(n, d) \rightarrow \cdots \rightarrow J^{0}(n, d) \rightarrow \mathbb{R}^{n} .
$$

Corollary 3. The map germ $X \rightarrow X^{(0)}$ from an integral submanifold to the graph factors through the normalization map $X=\mathbb{R}^{n} \rightarrow \Gamma_{m}^{n}$ provided that the projection $X \rightarrow \mathbb{R}^{n}$ to the base is Whitnean. In particular the graph is the image of a suitable $\operatorname{map} \Gamma_{m}^{n} \rightarrow J^{\prime}(n, d)$.

Once we found graphs of our multiple-valued functions to be images of $\Gamma_{m}^{n}$ we can try to reformulate all properties of the functions in terms of the graphs only and avoid mentioning integral submanifolds. At first we may assume $\Gamma_{m}^{n}$ to be embedded into a bigger bundle, say $J^{0}(n, d+n)$, and then projected onto the graph in $J^{0}(n, d)$ along an affine subbundle. For any such a subbundle (or better to say for any embedding $\Gamma_{m}^{n} \hookrightarrow J^{0}(n, d+n)$ over the standard projection $\left.\Gamma_{m}^{n} \rightarrow \mathbb{R}^{n}\right)$ the image of $\Gamma_{m}^{n}$ in $J^{0}(n, d)$ can be considered as a graph and recovers some integral $X$ in $J^{m}(n, d)$ (since smooth functions of $m$ times differentiable functions are $m$ times differentiable) but this $X$ can happen to be singular.

Lemma 2. The integral variety $X$ is singular if and only if

$$
\left(p_{i}^{(0, \ldots, 0, m)}\right)^{\prime}(0,0)=0, i=1, \ldots, d .
$$

Proof. The integral variety $X$ is the image of the non-singular integral submanifold in $J^{m}(n, d+n)$ under a suitable projection. $X$ is singular if and only if the projection has degenerate differential at the origin. The differential of the Whitney mapping at 
the origin has one-dimensional kernel generated by $\partial / \partial x$. Therefore the criticality condition is $\partial p_{i}^{\alpha} / \partial x=0$ at the origin for all $i$ and $\alpha$. From Lemma 1 we have

$$
\left.\frac{\partial}{\partial x}\right|_{(0,0)} p^{\alpha}=\left.\frac{\partial^{|\alpha|}}{\partial q^{\alpha}}\right|_{(0,0)}\left(p^{(0, m)}\right)^{\prime} \frac{F^{m}}{m !}
$$

since $\partial q_{n} / \partial x=0$ at the origin. Therefore for $\left|\alpha_{n}\right|<m$ the derivatives vanish automatically and the only criticality condition left is that with $\alpha=(0, \ldots, 0, m)$.

The criticality condition of Lemma 2 can be reformulated geometrically as the property of the subspace $T^{n+1}$ to have non-trivial intersection with the fiber of the projection $J^{0}(n, d+n) \rightarrow J^{0}(n, d)$. This completes the proof of Theorem 2 .

\section{$\S 5$ BRINGING TO NORMAL FORMS}

Let us consider a multiple-valued vector-function given by its integral submanifold $X \subset J^{m}(n, d)$ with $d \geqslant n$ at a point where the projection $X \rightarrow \mathbb{R}^{n}$ has Whitney singularity of "degree" $\mathrm{n}$. The graph of this vector-function is locally the image of a map $\Gamma_{m}^{n} \rightarrow J^{0}(n, d)$. According to Theorem 2, perturbing the map locally we also perturb the integral submanifold $X$. Since $2 n \leqslant n+d$ a generic perturbation has non-degenerate differential $T^{2 n} \rightarrow \mathbb{R}^{n+d}$. Therefore the graph of such a perturbation is locally isomorphic to $\Gamma_{m}^{n}$.

In order to normalize the position of the graph with respect to a coordinate system on the bundle $J^{0}(n, d) \rightarrow \mathbb{R}^{n}$ we first normalize the Whitney map $X \rightarrow$ $\mathbb{R}^{n}$ and then invoke the 2-nd part of Theorem 1. According to it the coordinate functions $\left(q_{1}, \ldots, q_{n}, P_{1}, \ldots, P_{d}\right)$ on $J^{0}(n, d)$ can be expressed through the canonical coordinates $\left(q_{1}, \ldots, q_{n}, p_{1}, \ldots, p_{n}\right)$ of the Lagrangian model as linear combinations

$$
P_{i}=A_{i 1}(q) p_{1}+\cdots+A_{i n}(q) p_{n}+Q_{i}(q)
$$

where the $d \times n$-matrix $\left(A_{i j}\right)$ has rank $n$ at the origin. This means that a suitable affine automorphism of the bundle $(q, P) \mapsto q$ brings the coordinate system to the normal form

$$
P_{1}=p_{1}, \ldots, P_{n}=p_{n}, P_{n+1}=\cdots=P_{d}=0 .
$$

Let us now consider the case $d=1$ of scalar multiple-valued functions. What we have learned is that in a suitable coordinate system $\left(q_{1}, \ldots, q_{n}, u\right)$ on $J^{0}(n, 1)$ the multiple-valued function $u=u(q)$ is the function of critical values in the following family of functions of one variable, $x$, :

$$
\int_{0}^{x} \phi(y, q) F_{n}^{m}(y, q) d y+Q(q)
$$

where $\phi(0,0) \neq 0$ according to Lemma 2. Changing the sign of $u$ if necessary we may assume $\phi$ to be positive.

Now we invoke the versality theorem for fractional forms in order to bring the family of 1-forms $\phi F_{n}^{m} d x$ to the normal form $F_{n}^{m} d x$ by a fibered change of the variable $x$ and parameters $q$. Since the potential of a 1-form and its critical values are uniquely determined by the form up to a constant summand, the same change of variables brings the multiple-valued function $u$ to the required normal form plus a regular function of the parameters $q$. Thus in order to normalize the singularity 
we have not even used linear transformations of the "dependent variable" $u$ but only change of its sign, changes of "independent variables" $q$ and subtraction of a regular function.

In the end let us consider the more general case where the number $N$ of independent variables exceeds the index $n$ of the Whitney singularity. This means that the projection $X^{N} \rightarrow \mathbb{R}^{N}$ has (a suspension of) the Whitney singularity along an $N-n$-dimensional submanifold $Y \subset \mathbb{R}^{N}$. Fibering $\mathbb{R}^{N}$ over $Y$ we may consider $Y$ as a space of additional parameters. Bringing the multiple-valued functions to the normal forms is based now on the obvious observation that all our previous constructions bear such a dependence on additional parameters.

In particular our perturbation - to - a - general - position argument still works at generic points of $Y$ and completes the proof. Notice that for $d \geqslant n$ "non-generic"

points form a subset of codimension one in $Y$ and classification of the degeneracies of multiple-valued vector-functions near such points remains an open problem.

I am thankful to V.I.Arnold who pointed to me some recent papers [5],[14],[15] on singularities in PDE.

\section{REFERENCES}

1. V. I. Arnold, Normal forms of functions near degenerate critical points, Weyl groups $A_{k}, D_{k}$, $E_{k}$ and Lagrangian singularities, Funct. Anal. Appl. 6 (1972), no. 4, 3-25.

2. V. I. Arnold, Potential flows of collisionless particles, Lagrangian singularities and metamorphoses of caustics, Trudy seminara im. Petrovskogo 8 (1982), 5-53. (Russian)

3. V. I. Arnold, Lagrangian manifolds with singularities, asymptotical rays and open swallowtail, Funct. Anal. Appl. 15 (1981), no. 4, 1-14.

4. V. I. Arnold, A. N. Varchenko, S. M. Gusein-Zade, Singularities of differential maps, vol. 1, Birkhäuser, Boston, 1985.

5. R.E.Caflisch, N.Ercolani, T.Y.Hou, E.Landis, Multi-valued solutions and branch point singularities for non-linear hyperbolic or elliptic systems, Commun. Pure and Appl. MAth. 46 (1993), 453-499.

6. A. B. Givental, Singular Lagrangian varieties and their Lagrangian mappings, Sovrem. probl. matematiki. Noveishie dostizheniya, vol. 33, VINITI AN SSSR, Moscow, 1988, pp. 55-112; English transl. in J. of Soviet Math. (1990) pp. 3246-3278

7. A. B. Givental, Varieties of polynomials having a root of fixed co-multiplicity and generalized Newton equation, Funct. Anal. Appl. 16 (1982), no. 1, 13-18.

8. A. B. Givental, Singular Lagrangian varieties and irreducible $s_{2}$ - modules, Russian math. surveys 38 (1983), no. 6, 109-110.

9. M. Golubitsky, V. Guillemin, Stable mappings and their singularities, Springer-Verlag, NewYork, 1973.

10. V. P. Kostov, Versal deformations of differential forms of degree $\alpha$ on the straight line, Russian math. surveys 40 (1985), no. 5, 235-236.

11. S. K. Lando, Normal forms of powers of volume forms, Funct. Anal. Appl. 19 (1985), no. 2, 78-79.

12. V. V. Lychagin, Geometrical singularities of solutions of nonlinear differential equations, Dokl. Akad. Nauk. SSSR 261 (1981), no. 6, 1299-1303. (Russian)

13. B. Morin, Formes canoniques des singularités d'une application différentiable, Compt. Rend. Acad. Sci. (Paris) 260 (1965), 5662-5665.

14. A.Kh.Rakhimov, Singularities of solutions of quasilinear equations, Algebra and Analysis 4 (1992), no. 4, 217-224.

15. A.Kh.Rakhimov, Singularities of Riemann invariants, Funct. Anal. Appl. 27 (1993), no. 1, 46-59.

16. V. M. Zakalyukin, A generalization of Lagrangian triads, Usp. Mat. Nauk 40 (1986), no. 4, 180. (Russian)

Dept. of Mathematics, UC Berkeley, CA 94720, USA

E-mail address: givental@math.berkeley.edu 